\title{
Desenvolvimento e comparação de um método de construção de arcos ortodônticos individualizados com um método tradicional de escolha de arcos ortodônticos pré-fabricados
}

\author{
Antonio José Borin Neto*, Rodrigo Cecanho**, Helena Cristina Francisco Pereira da Silva***
}

\section{Resumo}

Introdução: a existência de pacientes com arcos dentários e anatomias diferentes dos diagramas de arcos ortodônticos preestabelecidos justifica este trabalho. Objetivo: foi proposto mostrar um método de construção de arcos ortodônticos que respeitasse a anatomia individual de cada paciente e que fosse construído sem a necessidade de diagramas impressos pré-desenhados. Métodos: em vinte modelos inferiores de gesso, foram colados, no centro da coroa clínica dos seus dentes, braquetes e tubos. Com o auxílio de um paquímetro digital associado a um aparelho que posicionou os modelos com o plano oclusal paralelo ao solo, foi possível medir a distância da ponta de cúspide e meio da face incisal de cada dente até o fundo da canaleta do respectivo braquete e tubo colado. De posse dessas medidas, foram realizadas impressões das cúspides dentárias dos modelos em uma lâmina de cera 7 e, a partir dessas cúspides, pontuou-se a cera usando a distância obtida (entre a ponta de cúspide/meio da incisal dos dentes e a canaleta do braquete/entrada do tubo). Esses novos pontos serviram de referência para a construção dos arcos ortodônticos individualizados. Resultados e conclusão: esse método de construção foi denominado "arcograma", o qual foi comparado com um método pré-conformado de diagramação ("Tru-arch"), sendo considerado melhor que esse no tocante à adaptação à anatomia individual do arco.

Palavras-chave: Diagrama. Arco dentário. Anatomia do arco.

\section{INTRODUÇÃO}

Os dentes dos seres humanos são dispostos entre si formando arcos, os quais diferem anatomicamente de indivíduo para indivíduo, acompanhando a forma do osso basal. Por isso, apresentam características individuais importantes para as funções fisiológicas às quais são submetidos. Análises estatísticas das dimensões dos arcos dentários em diferentes etnias mostraram que tais formas são controladas, preferencialmente, por fatores genéticos e têm menor influência ambiental ${ }^{16}$.

Várias pesquisas mostram como é a estabilidade

* Especialista em Ortodontia - Profis / Bauru. Mestre em Ortodontia - Centro de Pós-Graduação São Leopoldo Mandic. Professor da EAP/APCD - Ribeirão Preto/Franca e CIEDEF - órgão anexo aos departamentos de Periodontia e Cirurgia da FORP/USP e Cabeça e Pescoço do Hospital das Clínicas da FMRP/USP.

** Mestre e doutor em Anestesiologia - Farmacologia e Terapêutica - Faculdade de Odontologia de Piracicaba - UNICAMP. Professor Titular da Faculdade de Odontologia e Centro de Pós-Graduação São Leopoldo Mandic - Campinas /SP.

*** Mestre em Ortodontia pela São Leopoldo Mandic. 
das dimensões dos arcos dentários. Um estudo longitudinal durante o período de 45 anos de vida verificou que as distâncias intercaninos e intermolares aumentavam significativamente entre os 3 e os 13 anos de idade, tanto na maxila como na mandíbula e, depois da completa irrupção da dentadura permanente, ocorria uma pequena diminuição na largura dos $\operatorname{arcos} 5$. Outro estudo, utilizando 271 modelos de indivíduos com idades de 3 anos e 6 meses até 13,5 anos, constatou pouco crescimento lateral nos arcos na região dos primeiros molares permanentes ${ }^{7}$. Clinicamente, entretanto, pode ser considerado que as distâncias intercaninos e intermolares são estabelecidas aos 8 anos de idade, depois da irrupção dos incisivos, com algum aumento mínimo até a dentadura permanente se completar - o que ocorre por volta dos 13 anos de idade - e que ambas as larguras, mas principalmente a distância intercaninos inferiores, diminuem no período pós-tratamento, quando ocorre uma expansão durante a terapia ortodôntica, mesmo que essa seja mínima ${ }^{5}$. Portanto, quanto maior é o aumento nessa dimensão durante o tratamento, menor é a estabilidade após a finalização. Sendo assim, no tratamento ortodôntico, é fundamental manter-se a distância transversal intercaninos inferiores do arco original, como fator importante para se conseguir estabilidade em longo prazo $9,10,26,30$. Esse fato pode ser exemplificado por um trabalho que avaliou mudanças nos arcos inferiores de pacientes com Classe I, II, divisões 1 e 2, que sofreram tratamento ortodôntico e - após análise de 80 modelos inferiores nas fases pré-tratamento, final de tratamento e 10 anos pós-tratamento - concluiu-se que as larguras intercaninos caminharam em direção às dimensões iniciais. Além disso, o comprimento do arco diminuiu em todos os pacientes e a largura intermolares apresentou uma redução maior nos casos tratados com extrações dentárias ${ }^{26}$.

Em relação à forma dos arcos, essas parecem se comportar também como as dimensões transversais, ou seja, as mudanças introduzidas no tratamento não foram estáveis em cerca de 70\% dos $\operatorname{casos}^{10,11,18,22,30}$. Portanto, não se deve esperar que uma forma de arco se encaixe em todos os arcos dentários e que adaptações individuais são necessárias para que se obtenham resultados ortodônticos estáveis.

$\mathrm{Na}$ literatura ortodôntica, o estudo das formas e dimensões dos arcos dentários é um tema muito debatido e controverso, foram usados recursos matemáticos e/ou geométricos, simples ou complexos $^{8,9,12}$, procurando - por meio de curvas perfeitas - atingir a diversidade natural encontrada na anatomia e fisiologia humana, e suas adaptações morfológicas solicitadas pelas funções neurovegetativas e pela neuromusculatura. Portanto, os arcos ortodônticos fabricados oriundos dessa filosofia são representados por curvas simétricas, obtidas por meio de fórmulas matemáticas ${ }^{9,24,27,29}$, as quais, na maioria dos casos não são coincidentes com a forma e com o tamanho dos arcos naturais ${ }^{4}$, sendo que a utilização de tais arcos levaria à padronização de todos os arcos dentários durante o tratamento ortodôntico, não respeitando suas individualidades e provocando, portanto, instabilidade pós-tratamento.

\section{OBJETIVO}

O objetivo deste trabalho foi demonstrar um método de construção de arcos ortodônticos individualizados ("arcograma") comparando-o a um método tradicionalmente usado na escolha de arcos ortodônticos pré-fabricados ("Tru-arch").

\section{METODOLOGIA}

Amostra

A amostra constituiu-se de 20 modelos de arcos dentários inferiores (Fig. 1) de 11 indivíduos com Classe I de Angle, 6 com Classe II e 3 com Classe III, com todos os dentes permanentes presentes - com exceção dos terceiros molares -, apinhamento dentário leve ou moderado na região anterior e todos os dentes bem posicionados na base óssea. Desses, 16 eram do gênero feminino 
e 4 do gênero masculino, com idade mínima de 11,9 anos e máxima de 22,5 anos, provenientes do arquivo particular do pesquisador.

O estudo foi desenvolvido em três etapas:

1) Obtenção da média da distância entre a ponta de cúspide e a região média incisal ao centro da canaleta/entrada do tubo de todos os dentes dos modelos.

2) Demonstração da construção do "arcograma".

3) Comparação entre as dimensões transversais dos arcos ortodônticos construídos por meio dos "arcogramas" da amostra com uma marca comercial de arco ortodôntico tradicional pré-fabricado ("Tru-arch").

$1^{\text {a }}$ etapa - obtenção da média da distância entre as pontas de cúspides ou região média incisal ao centro das canaletas dos braquetes ou tubos para construção do "arcograma"

Para se obter a média da distância entre as pontas das cúspides dos molares, pré-molares e caninos e o meio das faces incisais dos incisivos inferiores ao centro das canaletas dos braquetes e entradas dos tubos molares, foi definido, inicialmente, o centro da coroa clínica. Para isso, as coroas dentárias foram medidas nos sentidos mesiodistal e oclusocervical (ou incisocervical) com paquímetro digital (Marberg ${ }^{\circledR}$ ) com grau de precisão de $0,01 \mathrm{~mm}$ e divididas ao meio com um traço horizontal e outro vertical, realizados com o auxílio de uma lapiseira com grafite $0,5 \mathrm{~mm}$ (Fig. 2). A intersecção destas retas mostra o centro da coroa ${ }^{2}$, local aonde foram colados braquetes e tubos ortodônticos da técnica de Roth com canaleta 0,022" x 0,028" (Ovation - GAC International Inc. ${ }^{\circledR}, \mathrm{NY} /$ USA) por meio de adesivo instantâneo (éster de cianoacrilato) (Fig. 3).

Para as medições da distância de interesse (pontas de cúspides/meio da incisal dos dentes ao fundo das canaletas/entradas dos tubos colados nos respectivos dentes), foi utilizado um instrumento específico, idealizado pelo pesquisador, denominado "Máquina de medição" (Fig. 4), composta de um suporte para um paquímetro digital Marberg (1), preso a um cilindro vertical (2) fixado em uma base de aço (3), com molas de apoio (4) e um parafuso para estabilização (atrás do suporte) para controlar sua altura na posição necessária, sobre os dentes. Cada modelo inferior foi posicionado e fixado com o plano oclusal paralelo ao solo, após desgaste da base do modelo quando necessário, sobre uma pequena mesa de metal (5) que desliza sobre a base (Fig. 5), facilitando o posicionamento do modelo junto às pontas do paquímetro. Essas foram modificadas para o objetivo idealizado, do seguinte modo: a ponta anterior (A) foi preparada para receber um componente denominado "camisa de medição", que se movimenta verticalmente e possui uma lâmina que se posiciona dentro das canaletas dos braquetes ou entradas dos tubos dos respectivos dentes, mantendo um paralelismo com a parte posterior fixa (B), afinada para adaptação sobre as pontas de cúspides e meio das faces incisais dos incisivos inferiores (Fig. 6).

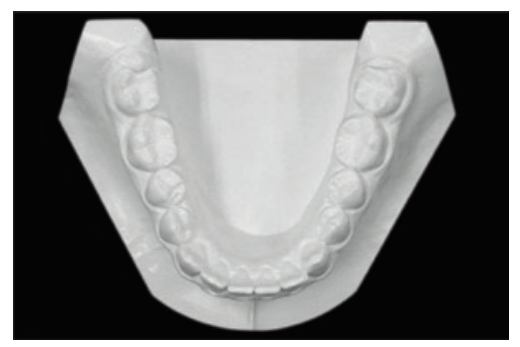

FIGURA 1 - Modelo do arco representativo.

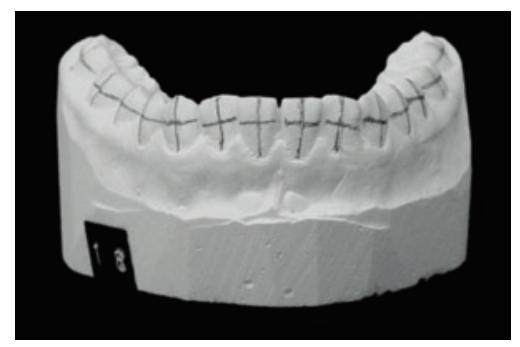

FIGURA 2 - Centros das coroas clínicas.

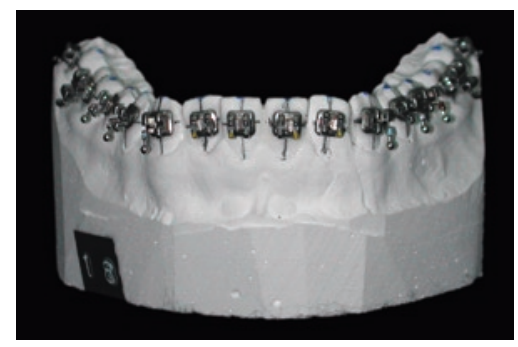

FIGURA 3 - Braquetes colados. 


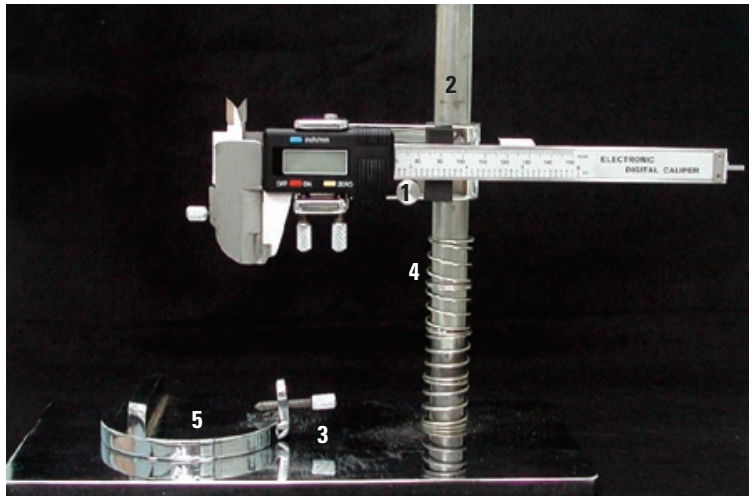

FIGURA 4 - Máquina de medição: 1) suporte do paquímetro, 2) cilindro vertical, 3) base de aço, 4) molas de apoio e 5) mesa para fixar modelo.

Foram, então, realizadas as medições das pontas de cúspides ou centro das superfícies incisais dos dentes até o centro das respectivas canaletas dos braquetes ou entrada dos tubos colados (Fig. 7, 8).

Após medidas as distâncias relacionadas a todos os dentes do arco inferior, foram calculadas as médias desses valores para cada dente do arco (Tab. 1). Uma vez que o desvio-padrão dessas médias foi considerado pequeno, optou-se por utilizar as mesmas com uma casa decimal de aproximação e fixou-se os valores por grupos de dentes, como padrão para qualquer modelo a ser utilizado futuramente, não havendo necessidade de se realizar tais medidas individualmente.

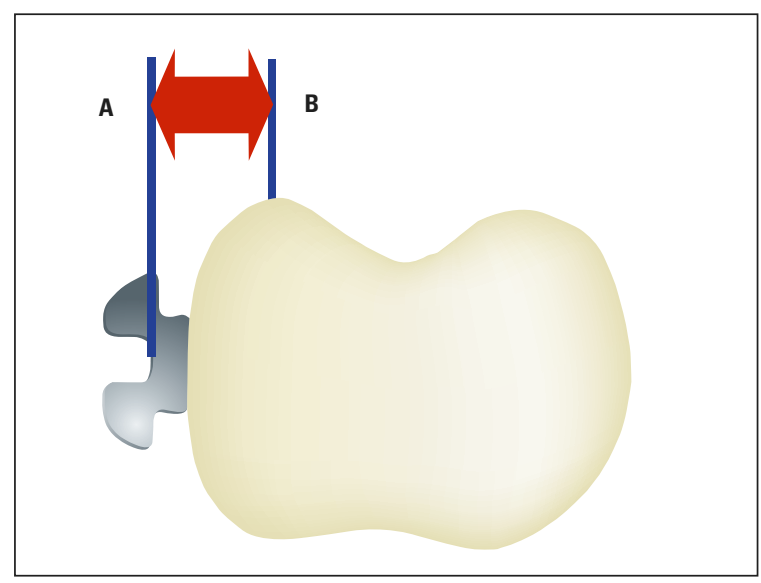

FIGURA 7 - Espaço medido entre o dente e o centro do braquete (A - B).

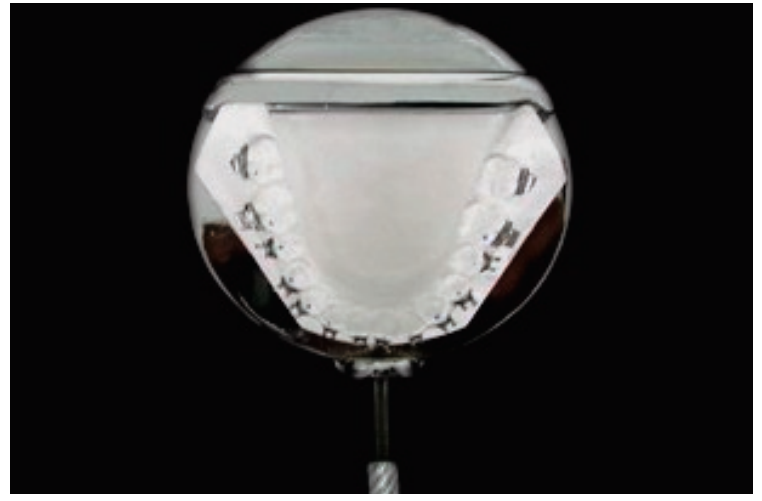

FIGURA 5 - Modelo fixado na mesa.

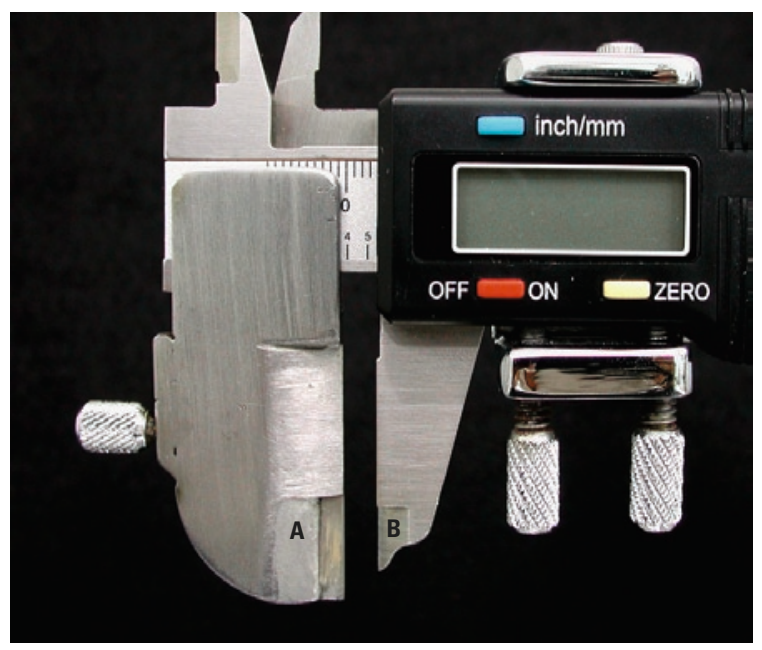

FIGURA 6 - Pontas modificadas do paquímetro (A, B).

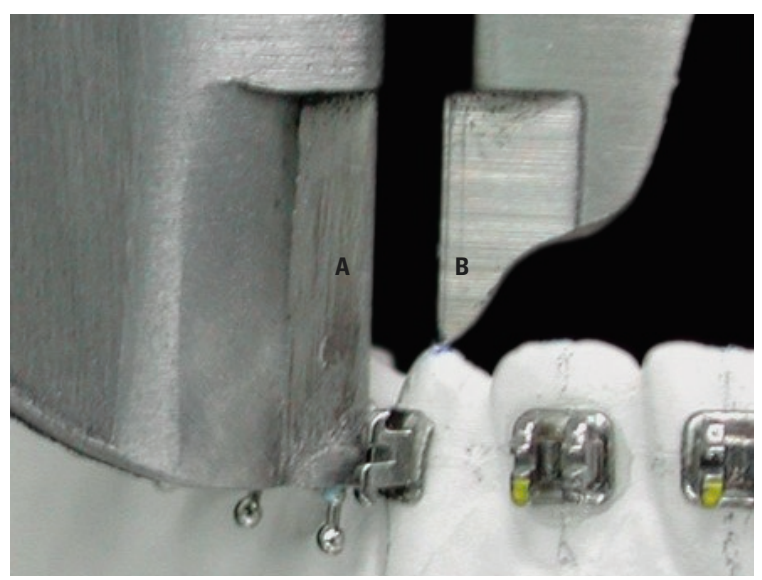

FIGURA 8 - Espaço medido pelo paquímetro entre o dente e o centro do braquete $(\mathbf{A}-\mathbf{B})$. 


\section{$2^{\text {a }}$ etapa - construção do "arcograma"}

Foram marcadas as pontas das cúspides vestibulares e os pontos médios das superfícies incisais nos modelos de gesso do arco dentário inferior ${ }^{8,12}$ (Fig. 9). Sobre o modelo foi pressionada uma lâmina de cera $n^{\circ} 7$ (Wilson ${ }^{\circledR}$, Polidental, Cotia/SP), tomando-se o cuidado de não perfurá-la, obtendose um molde das faces oclusais dos dentes (Fig. 10).

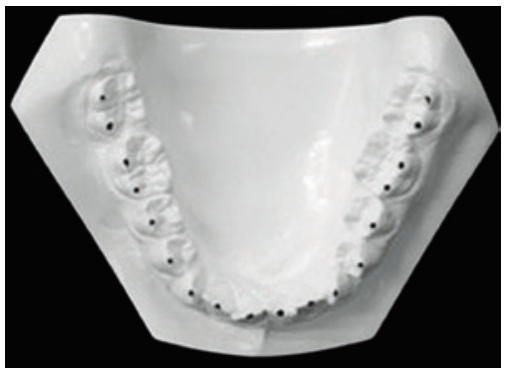

FIGURA 9 - Marcação dos pontos no modelo.

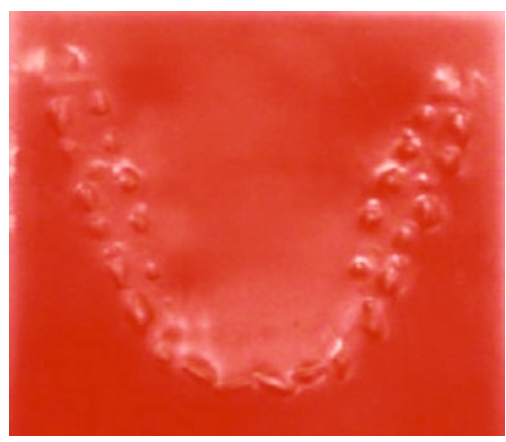

FIGURA 11 - Lâmina de cera removida sem distorções.
A lâmina de cera foi cuidadosamente removida do modelo, para evitar distorções (Fig. 11), e, aproveitando-se da sua translucidez, os pontos do modelo foram transferidos para a cera com a mesma caneta. Esses pontos foram denominados "pontos dentários" (Fig. 12).

Outro grupo de pontos foi marcado vestibularmente aos "pontos dentários". Esse grupo foi

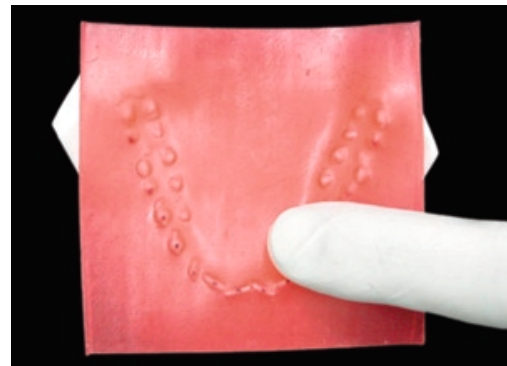

FIGURA 10 - Impressão das faces oclusais na lâmina de cera.

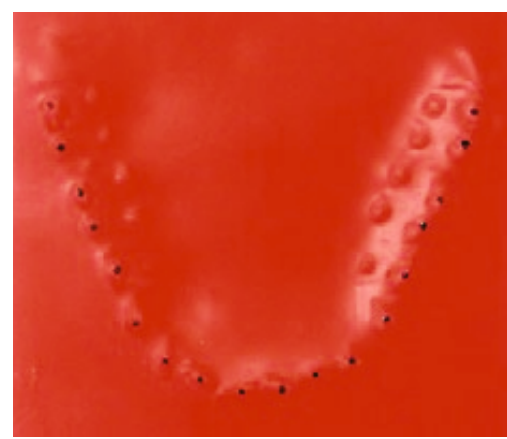

FIGURA 12 - Marcação dos "pontos dentários".

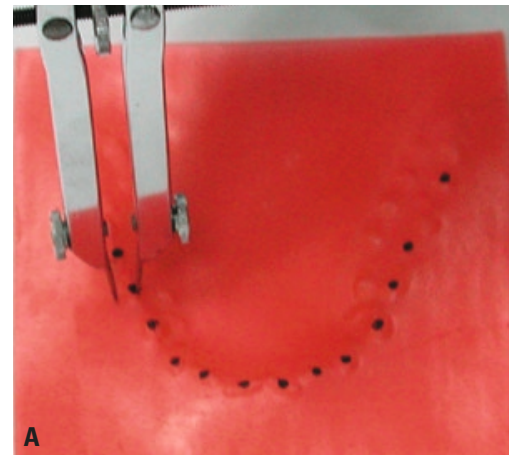

FIGURA 13 - Marcação dos "pontos do arco".

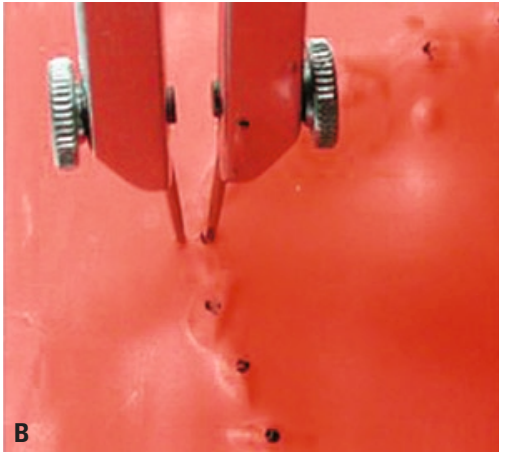

FIGURA 14 - Pontos do arco: "pontos principais" marcados na cor preta, "pontos intermediários" marcados na cor vermelha. 
chamado de "pontos do arco" (Fig. 13) e dividido em: "pontos principais" e "pontos intermediários" (Fig. 14). Os "pontos principais" foram marcados vestibularmente aos "pontos dentários", com o auxílio de um compasso de pontas secas, obedecendo-se, para cada dente, a média anteriormente obtida entre a ponta de cúspide e o centro da canaleta do braquete ou entrada do tubo: $1,5 \mathrm{~mm}$ para incisivos, $2,5 \mathrm{~mm}$ para caninos e $3 \mathrm{~mm}$ para pré-molares e molares (Tab. 1). Os "pontos intermediários" foram marcados no ponto médio entre os "pontos principais". Sua função foi aumentar a quantidade de pontos, para melhor definição do formato do arco.

Caso algum dente ou grupo de dentes se encontrasse fora da base óssea, pelo fato de estarem conectados pelo osso basal ${ }^{29}$, utilizou-se como "pontos dentários" apenas os dentes melhores situados no arco dentário, ignorando-se aqueles excêntricos à forma do arco. Para melhor estabelecer as dimensões transversais, pode-se utilizar alguns parâmetros auxiliares na construção do arco: a borda WALA ${ }^{3}$, para molares inferiores, definida como sendo o limite inferior da gengiva inserida, a qual, segundo o autor, serve para avaliar a posição do $1^{\circ}$ molar inferior, relacionando-a ao ponto EV (eixo vestibular da coroa clínica) - um molar bem posicionado tem seu ponto EV a $2 \mathrm{~mm}$ da borda WALA (Fig. 15). Quanto à distância intercaninos inferiores, deve-se considerar as pontas de cúspides do modelo inicial (Fig. 16), sugerido pela maioria dos pesquisadores e devidamente corrigido caso esses dentes estejam inclinados ou angulados ${ }^{6}$. Alternativamente, pode-se usar como referência para a distância intermolares inferiores a distância entre as fossas centrais dos molares superiores corrigidas por meio de análise própria para modelos superiores ${ }^{19}$ e relacioná-las com as cúspides inferiores em largura (Fig. 17).

Com os "pontos do arco" (principais e intermediários), formata-se o "arcograma" (Fig. 18) e sua individualização (correção) deve ser realizada levando-se em consideração os objetivos do tratamento, o planejamento das correções necessárias,

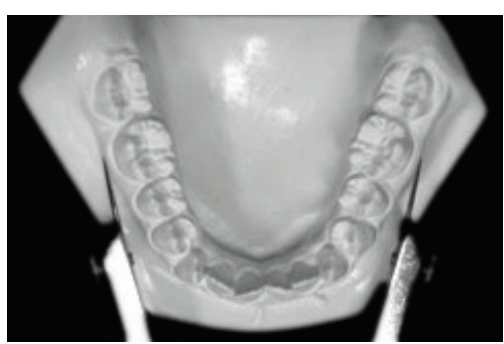

FIGURA 15 - Medida da distância transversal (borda WALA).

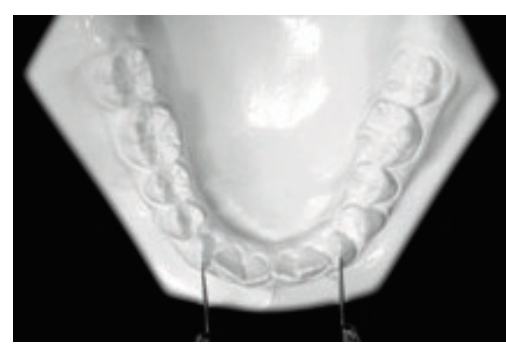

FIGURA 16 - Medida da distância intercaninos no modelo.

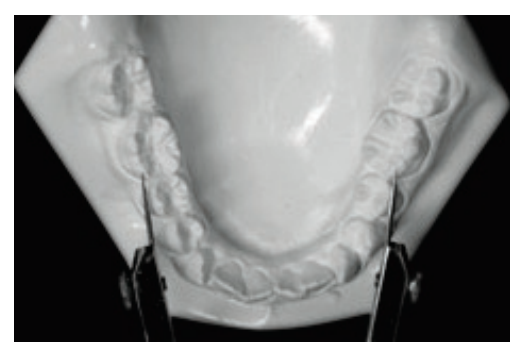

FIGURA 17 - Medida da distância intermolares no modelo.

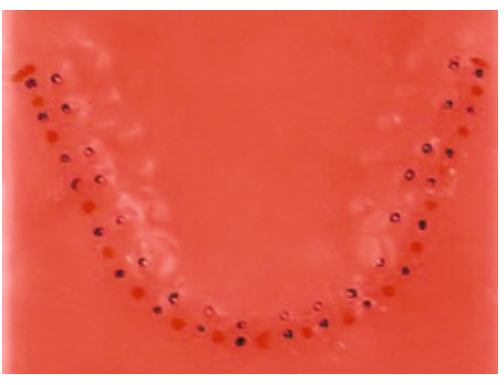

FIGURA 18 - "Arcograma" formatado.

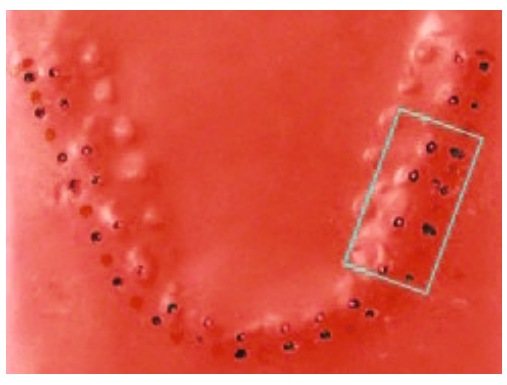

FIGURA 19 - "Arcograma" individualizado (corrigido) e finalizado. 
os posicionamentos individuais de dentes e de grupos de dentes e as dimensões transversais da base óssea. Para isso, deve-se corrigir, a partir dos "pontos dentários", expandindo ou contraindo, posicionando para anterior ou para posterior os "pontos principais" e complementar a formatação do arco corrigido, com os "pontos intermediários". Assim, finaliza-se o "arcograma" (Fig. 19).

$3^{a}$ etapa - comparação entre as dimensões transversais dos arcos ortodônticos construídos nos "arcogramas" da amostra com o arco ortodôntico tradicional pré-fabricado ("Truarch")

Para efeito de comparação entre o arco ortodôntico construído por meio da técnica do "arcograma" e uma marca de arco ortodôntico pré-fabricado de uso tradicional, denominado
"Tru-arch", foi confeccionado um arco ortodôntico com fio de aço de NiCr 0,019" x 0,025" ("A" Company - Ormco Corporation, USA) mostrado na figura 20, formatado no "arcograma" para cada modelo da amostra. O arco "Tru-arch" de níquel titânio (NiTi) escolhido, também fabricado pela "A" Company - Ormco Corporation, USA, foi o de tamanho médio 0,019" x 0,025".

Em seguida, foram selecionadas as seguintes distâncias nos arcos construídos por meio do "arcograma": intermolares, formada pelos pontos do "arco" correspondentes às cúspides mesiovestibulares dos primeiros molares inferiores; distância interpré-molares, medida por meio dos "pontos intermediários" entre os "pontos principais" correspondentes às cúspides vestibulares, dos primeiros e segundos pré-molares inferiores; e a distância entre os "pontos principais" das cúspides vestibulares dos
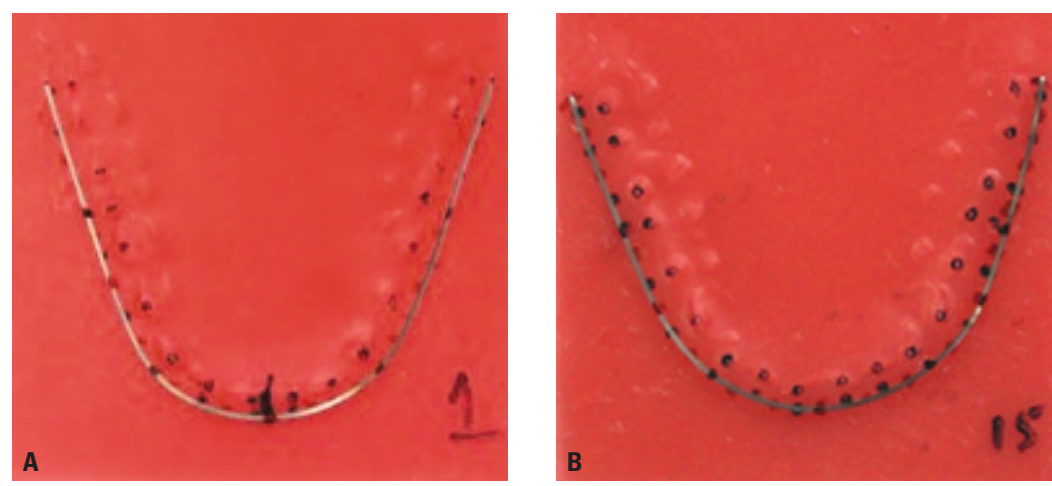

FIGURA 20 - Arcos ortodônticos: A) NiCr formatado por meio da técnica do "arcograma" e B) NiTi "Truarch", ambos 0,019" x 0,025".
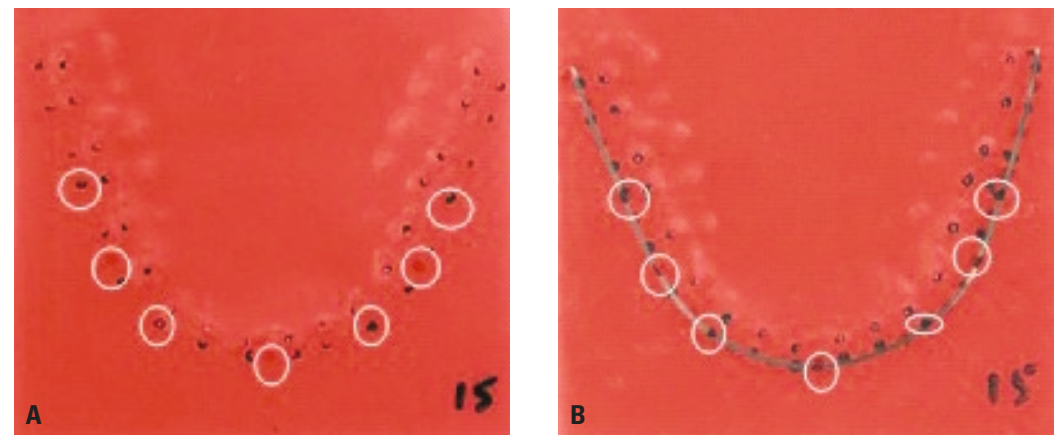

FIGURA 21 - A) Círculos assinalando os pontos selecionados para medição das distâncias intermolares, interpré-molares e intercaninos; B) arco NiCr individualizado construído no "arcograma" com pontos selecionados.

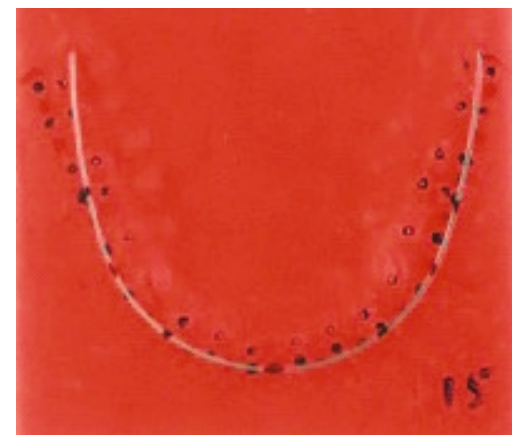

FIGURA 22 - Arco tradicional "Tru-arch" (NiTi) sobre 0 "arcograma" com pontos selecionados. 
caninos inferiores direito e esquerdo, que representou a distância intercaninos. A linha média do arco foi determinada como sendo o "ponto intermediário" entre os "pontos principais" dos incisivos centrais inferiores e foi utilizada como referência para o posicionamento dos arcos em estudo. Todos esses pontos foram marcados nos arcos ortodônticos de $\mathrm{NiCr} 0,019$ " x 0,025" devidamente adaptados sobre o respectivo "arcograma", e da mesma forma para os arcos de NiTi tamanho médio inferiores denominados "Tru-arch" (Fig. 21, 22).

Em seguida, as medidas intermolares, inter-prémolares e intercaninos foram realizadas com um compasso de pontas secas (0 - 369, Dentaurum, Alemanha), no "arcograma" e no modelo tradicional "Tru-arch" em cada modelo da amostra (Fig. 23-28). As distâncias foram representadas como médias e desvios-padrão e as médias de cada distância foram comparadas entre os grupos "arcograma" e "Tru-arch", por meio do teste de Wilcoxon.

\section{RESULTADOS}

Os resultados obtidos para a distância da ponta de cúspide e incisal até o centro do braquete e entrada do tubo - que representa, na formatação do "arcograma", a região onde o arco ortodôntico é construído - estão representados na tabela 1 .

As dimensões transversais nas regiões dos arcos ortodônticos construídos por meio do "arcograma" e dos arcos pré-fabricados ("Tru-arch") mostraramse estatisticamente diferentes após a aplicação do teste de Wilcoxon $(\mathrm{p}<0,05)$ na região interprémolares e intermolares (Tab. 2).

\section{DISCUSSÃO}

Por mais de cem anos, estudos têm sido realizados para definir a forma ideal do arco dentário, frequentemente usando o conceito de que o arco natural é simétrico e pode ser representado por uma curva perfeita derivada de fórmulas geométricas, algébricas e aritméticas ${ }^{9,24,27,29}$.

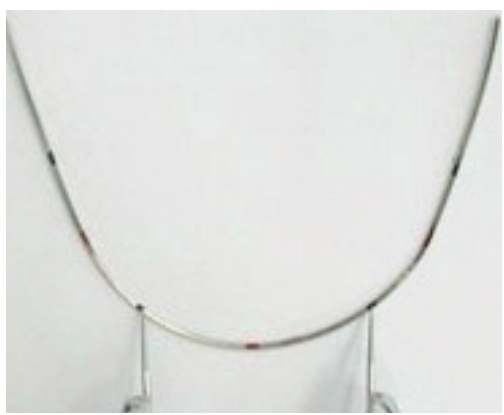

FIGURA 23 - Distância intercaninos ("arcograma").

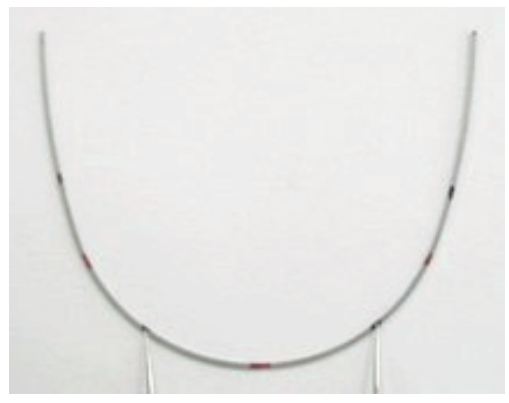

FIGURA 26 - Medição da distância intercaninos, arco NiTi ("Tru-arch").

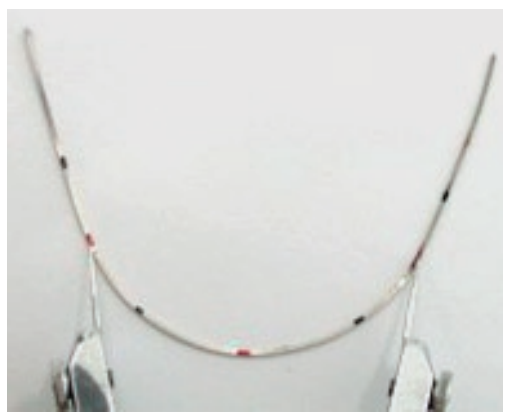

FIGURA 24 - Medição da distância interpré-molares ("arcograma").

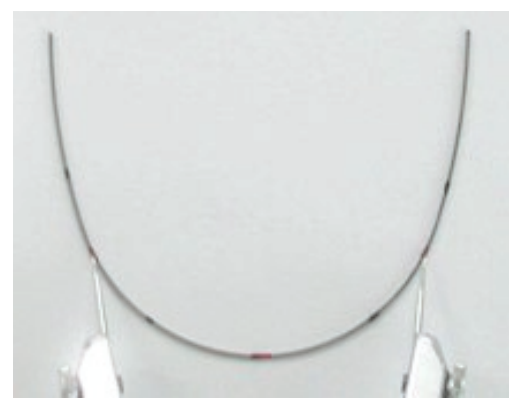

FIGURA 27 - Medição da distância interpré-molares, arco NiTi ("Tru-arch").

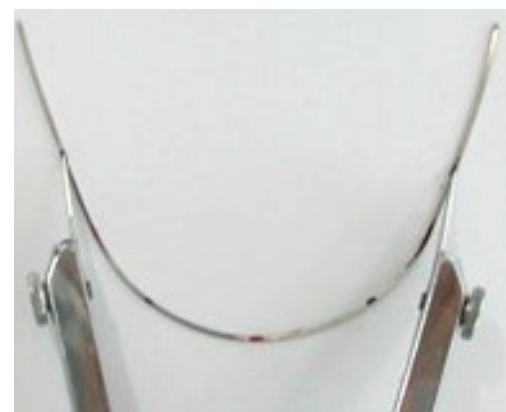

FIGURA 25 - Medição da distância intermolares ("arcograma").

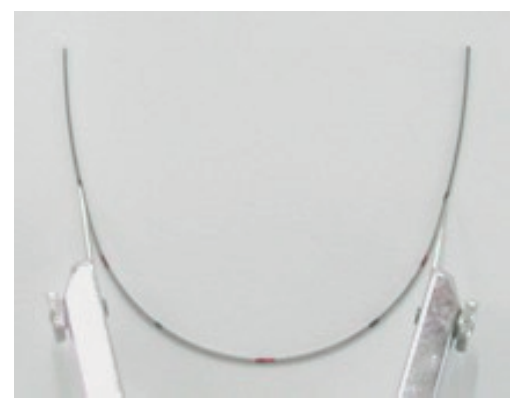

FIGURA 28 - Medição da distância intermolares, arco NiTi ("Tru-arch"). 
Entre essas formas investigadas, incluem-se a elipse, a parábola e a curva catenária, como também matemáticas tais quais, sessão cônica, função polinomial e "cubic spline". Cada uma dessas formas de arco tem sido acolhida, como também criticada, pelos ortodontistas. Porém, as formas de arcos

TABELA 1 - Média, desvio-padrão e aproximação dos valores referentes à distância da ponta de cúspide/incisal à canaleta do braquete/tubo, de 20 modelos de gesso inferiores estudados.

\begin{tabular}{|c|c|c|c|}
\hline dente & $\begin{array}{l}\text { média } \\
(\mathrm{mm})\end{array}$ & $\begin{array}{l}\text { desvio-padrão } \\
\text { (mm) }\end{array}$ & $\begin{array}{c}\text { aproximação } \\
(\mathrm{mm})\end{array}$ \\
\hline 47 & 2,9 & 0,4 & 3,0 \\
\hline 46 & 3,1 & 0,2 & 3,0 \\
\hline 45 & 3,1 & 0,3 & 3,0 \\
\hline 44 & 3,0 & 0,3 & 3,0 \\
\hline 43 & 2,6 & 0,3 & 2,5 \\
\hline 42 & 1,7 & 0,4 & 1,5 \\
\hline 41 & 1,5 & 0,3 & 1,5 \\
\hline 31 & 1,6 & 0,3 & 1,5 \\
\hline 32 & 1,6 & 0,3 & 1,5 \\
\hline 33 & 2,5 & 0,2 & 2,5 \\
\hline 34 & 2,9 & 0,3 & 3,0 \\
\hline 35 & 3,0 & 0,2 & 3,0 \\
\hline 36 & 3,1 & 0,3 & 3,0 \\
\hline 37 & 2,9 & 0,3 & 3,0 \\
\hline
\end{tabular}

ortodônticos produzidas comercialmente, e utilizadas na atualidade, derivam de tais fórmulas.

Diante da constatação das recidivas, que ocorrem pós-tratamento ortodôntico, na forma de apinhamentos e/ou redução nas dimensões dos arcos dentários ${ }^{4,6,10,11,18,26,29,30}$, estimula-se a procura por métodos para construção de arcos ortodônticos individualizados, baseando-se nas formas originais dos arcos dentários tratados. Essa proposta tem sido acolhida com generosidade pela comunidade ortodôntica, no entanto as novas abordagens metodológicas continuam, na sua maioria, utilizando-se de diagramas em formas de cartões - usados por meio da seleção de um entre vários (aquele que melhor se adapta à forma do arco dentário) e sendo o definido como o diagrama "perfeito" para a construção dos arcos ortodônticos do início ao final do tratamento 6,13,14,17,22. Tais métodos não podem adaptar-se a todas as formas e dimensões de arcos e ainda não levam em conta a possibilidade da assimetria, frequentemente presente.

Atualmente, novos métodos propondo arcos ortodônticos de ligas de aço cada vez mais sofisticadas, com dimensões únicas, tanto para o arco superior como para o inferior, posicionando os dentes além das dimensões basais originais, vêm sendo utilizados com o intuito de evitar-se extrações dentárias na correção ortodôntica. No entanto, a literatura carece de estudos em longo prazo que mostrem estabilidade de tratamentos realizados com tais terapias e a mesma literatura é ampla em mostrar que quanto maiores são as alterações de forma e tamanho dos arcos dentários, durante o tratamento ortodôntico, maior é o

TABELA 2 - Diferenças das distâncias intercaninos, interpré-molares e intermolares entre o arco construído sobre o "arcograma" e o “Tru-arch".

\begin{tabular}{|c|c|c|c|c|c|}
\hline \multirow{2}{*}{ distância } & \multicolumn{2}{|c|}{ "arcograma" } & \multicolumn{2}{|c|}{ "Tru-arch" } & \multirow[b]{2}{*}{$\mathbf{p}$} \\
\hline & média & d.p. & média & d.p. & \\
\hline intercaninos & 31,3250 & 1,8657 & 31,8500 & 2,0590 & 0,5919 \\
\hline interpré-molares & 44,5000 & 2,6458 & 46,5750 & 1,3306 & 0,0006 \\
\hline intermolares & 50,45 & 2,41104 & 52,65 & 1,13670 & 0,0007 \\
\hline
\end{tabular}

Teste: Wilcoxon $(5 \%, \mathrm{p}=<0,05)$. 
potencial de ocorrerem recidivas, tendenciando os arcos a retornarem às características originais ${ }^{4,6,9,10,}$ $11,18,28,29,30$.

O objetivo desse método foi construir um diagrama ortodôntico, que recebeu o nome de "arcograma", um método que por meio da distância entre as pontas de cúspides ou incisais e o fundo da canaleta dos braquetes ou tubos procura corrigir a forma do arco dentário, respeitando ao máximo as dimensões e a forma do osso basal. Para isso, foi desenvolvida uma metodologia que generalizou as distâncias anteriormente descritas (ponta de cúspide/incisal ao fundo das canaletas) para serem usadas em todos os pacientes, foi demonstrada passo a passo a técnica de confecção do "arcograma" e foi realizada uma comparação da adaptação desse com um método tradicionalmente utilizado, sendo o "arcograma" considerado melhor no quesito de adaptação aos arcos dentários naturais, pela diversidade, assimetria e individualidade desses últimos.

\section{CONCLUSÃO}

Este trabalho mostrou a viabilidade da utilização do "arcograma", um método de individualização da construção de arcos ortodônticos para a manutenção das características dimensionais e anatômicas do arco dentário.

Development and comparison of a individualized orthodontic arches construction method and a traditional method for choosing pre-manufactured orthodontic arches

\begin{abstract}
Introduction: The existence of exceptional anatomic dental arches justifies this work. Aim: It was proposed to show a orthodontic arches construction method that follow the individual anatomy of each patient, developed without any previous constructed diagram. Methods: Braces or molar tubes were bonded on 20 inferior natural cast models. With the aid of a digital paquimeter associated to an apparatus that positioned the occlusal planes parallel to the floor, the distance between the cuspid tip and the center of the slot of the brace and tube were measured. Impressions of the cuspids were made on a wax blade and the distance previously obtained were used to made a new conjunct of points vestibularly to the first marked points. These new points served as references to the construction of the individual orthodontic arch. Results and conclusion: The new method was named "arcogram" and it was compared to the Tru-arch pre-conformed arches, showing better adaptation to the arches anatomy.
\end{abstract}

Keywords: Diagram. Dental arch. Arch anatomy. 


\section{REFERÊNCIAS}

1. ANDREWS, L. F. Entrevista. Rev. Dental Press Ortodon. Ortop. Facial, Maringá, v. 2, n. 5, p. 6-8, set./out. 1997.

2. ANDREWS, L. F. Straight-wire: The concept and appliance. San Diego: L.A.Willes, 1989.

3. ANDREWS, L. F.; ANDREWS, W. A. The six elements of orofacial harmony. Am. J. Orthod. Orofacial Harmony, [S.I.], v. 1, no. 1, p. 12-34, Winter 2000.

4. ANTONIO, A. C. F. R.; ARAÚJO, M. T. S.; NOJIMA, L. I. Forma de arcada humana versus forma de arco níquel titânio. APEO: Rev. Ass. Paul. Esp. Ortod. Ortop. Facial, Piracicaba, v. 3, n. 1, p. 16-22, jan./ mar. 2005.

5. BISHARA, S. E.; JAKOBSEN, P. R.; TREDER, J. et al. Arch width changes from 6 weeks to 45 years of age. Am. J. Orthod. Dentofacial Orthop., St. Louis, v. 4, no. 111, p. 401-409, Apr. 1997.

6. CAPELOZZA, L. F.; CAPELOZZA, J. A. Z. Diagrama individual anatômico objetivo: uma proposta para escolha da forma dos arcos na técnica Straight-Wire, baseada na individualidade anatômica e nos objetivos de tratamento. Rev. Clin. Ortodon. Dental Press, Maringá, v. 3, n. 5, p. 84-92, out./nov. 2004.

7. COHEN, J. T. Growth and development of the dental arches in children. J. Am. Dent. Assoc., Chicago, v. 27, p. 1250-1260, 1940.

8. CURRIER, J. H. A computerized geometric analysis of human dental arch form. Am. J. Orthod., St. Louis, v. 56, no. 2 p. 164-179, Aug. 1969.

9. DAVIS, L. M.; BEGOLE, E. A. Evaluation of orthodontic relapse using the cubic spline function. Am. J. Orthod. Dentofacial Orthop., St. Louis, v. 113, no. 3, p. 300-306, Mar. 1998.

10. DE LA CRUZ, A. R.; SAMPSON, P.; LITTLE, R. M.; ARTUN, J.; SHAPIRO, P. A. Long-term changes in arch form after orthodontic treatment and retention. Am. J. Orthod. Dentofacial Orthop., St. Louis, v. 107, no. 5 p. 518-530, May 1995.

11. FELTON, J. M.; SINCLAIR, P. M.; JONES, D. L.; ALEXANDER, R. G. A computerized analysis of the shape and stability of mandibular arch form. Am. J. Orthod. Dentofacial Orthop., St. Louis, v. 92, no. 6, p. 478-483, Dec. 1987.

12. HNAT, W. P. ; FENDER, D. E.; LEGAN, H. L. The form of the human dental arch. Angle Orthod., Appleton, v. 68, no. 1, p. 29-36, Feb. 1998.

13. INTERLANDI, S. Diagrama para contorneamento do arco de canto individual. Rev. Assoc. Paul. Cir. Dent., São Paulo, v. 18, n. 1, p. 1-4, jan./fev. 1964.

14. INTERLANDI, S. Método para o traçado e emprego de um diagrama de contorneamento ortodôntico. Bol. Soc. Paul. Ortod., São Paulo, v. 4, n. 2, p. 5-9, jul./dez. 1966.

15. JONES, M. L.; RICHMOND, S. An assessment of the fit of a parabolic curve to pre and post-treatment dental arches. Br. J. Orthod., Oxford, v. 16, no. 2, p. 83-93, May 1989.

16. LAVELLE, C. L. B. A metrical study of dental arch form. J. Dent., Bristol, v. 6, no. 2, p. 120-124, June 1978.

17. LEITE, E. A.; PAIVA, M. G. Diagrama e arcos individualizados para aplicação em Ortodontia. Rev. Brás. Odontol., Rio de Janeiro, v. 25, n. 153, p. 287-294, set./out. 1968.

18. LITTLE, R. M. Stability and relapse of dental arch alignment. In: NANDA, R.; BURSTONE, C. J. Retention and stability in Orthodontics. Philadelphia: W. B. Saunders, 1993. p. 97-106.

19. MARTINS, S. F. Estudo comparativo entre as medidas obtidas por Korkaus, Schwarz e Índice de Pont, tomadas em amostras européias e as obtidas em uma amostra brasileira. 1993. Dissertação (Mestrado)-Universidade de São Paulo, São Paulo, 1993.

20. McCOY, J. D. A consideration of normal arch form and some of the methods of determining it. Int. J. Orthod., Lakewood, v. 5, no. 11, p. 697-723, Nov. 1919.

21. RABERIN, M.; LAUMON, B.; MARTIN, J.; BRUNNER, F. Dimensions and form of dental arches in subjects with normal occlusion. Am. J. Orthod., St. Louis, v. 104, no. 1, p. 67-72, July 1993.
22. ROBNETT, J. H. Segment concept in the arch pattern design. Am. J. Orthod. St. Louis, v. 77, no. 4, p. 355-367, Apr. 1980.

23. RUDGE, S. J. Dental arch analysis: arch form: a review of the literature. Eur. J. Orthod., Oxford, v. 3, no. 4, p. 279-284, 1981.

24. SAMPSON, P. D. Dental arch shape: a statistical analysis using conic sections. Am. J. Orthod., St. Louis, v. 79, no. 5, p. 535-548, May 1981

25. SAVOSTING-ASLING, I. The geometric analysis of mandibular dental arch form. Ann. Dent., New York, v. 39, no. 1, p. 3-11, Spring 1980.

26. SHAPIRO, P. A. Mandibular dental arch form and dimensiontreatment and post-retention changes. Am. J. Orthod., St. Louis, v. 66, no. 1, p. 58-70, July 1974.

27. SVED, A. Mathematics of the normal dental arch. Dental Cosmos, Philadelphia, v. 59, no. 11, p. 1116-1124, Nov. 1917.

28. TOIGO, E. C.; SOARES, L. H.; VIGORITO, J. W. Observações clínicas longitudinais das alterações pós-tratamento dos arcos dentários. Ortodontia, São Paulo, v. 28, n. 3, p. 4-12, set./dez. 1995.

29. WHITE, L. W. Individualized ideal arches. J. Clin. Orthod., Boulder, v. 12, no. 11, p. 779-787, Nov. 1978.

30. ZACHRISSON, B. U. Aspectos importantes da estabilidade a longo prazo. Rev. Dental Press Ortodon. Ortop. Facial, Maringá, v. 3, n. 4, p. 90-121, jul./ago. 1998.
Endereço para correspondência

Antonio José Borin Neto

Rua Rui Barbosa $n^{\circ} 1145$ sl. 42 / $4^{\circ}$ andar - Ed. Saint Moritz

CEP: 14.015-120 - Ribeirão Preto/SP

E-mail: borinneto@gmail.com 